\title{
The role of pancreatic insulin secretion in neonatal glucoregulation. II. Infants with disordered blood glucose homoeostasis
}

\author{
J M Hawdon, A Aynsley-Green, K Bartlett, M P Ward Platt
}

\begin{abstract}
Some neonates, such as those who are preterm or small for dates, become hypoglycaemic or hyperglycaemic. These disorders represent a failure of neonatal metabolic adaptation, but the underlying mechanisms are unclear. Data from studies of hypoglycaemic and hyperglycaemic infants were reviewed in the light of new data from studies of healthy neonates. Data from 28 neonates, who had disordered blood glucose homoeostasis, were analysed to determine the interrelationships between circulating concentrations of glucose, intermediary metabolites, glucagon and insulin, and glucose production rates.

Blood glucose concentrations ranged from 2.5 to $26.1 \mathrm{mmol} / \mathrm{h}$, and glucose production rates from 0 to $19 \cdot 2 \mathrm{mg} / \mathrm{kg} / \mathrm{min}$. Blood glucose concentrations were positively related to intravenous glucose infusion rates and to glucose production rates. A negative relationship existed between plasma glucagon and blood glucose concentrations, but there was a wide variation in plasma insulin levels at all blood glucose concentrations. No relationship between either plasma insulin or glucagon concentration and glucose production rate was shown.

It is concluded that in neonates with disordered blood glucose homoeostasis, blood glucose concentration is influenced by the rate of administration of glucose, with less precise internal control mechanisms than older subjects. This emphasises the importance of blood glucose monitoring and careful prescribing of exogenous glucose by clinicians caring for such infants.
\end{abstract}

(Arch Dis Child 1993;68:280-285)

Department of Child Health, University of Newcastle upon Tyne J M Hawdon

A Aynsley-Green

K Bartlett

M P Ward Platt

Correspondence to: Dr J M Hawdon, Institute of Child Health, Royal Liverpool Children's Hospital Alder Hey, Eaton Road,

Liverpool L12 2AP.

Accepted 25 September 1992

In adults, insulin secretion is increased and glucagon secretion is suppressed by high circulating blood glucose concentrations. In turn, these hormones modify the rate of hepatic glucose production, so that glucose production rates are modified in response to circulating blood glucose concentrations.

The newborn infant must also maintain blood glucose homoeostasis by changes in endogenous glucose production on cessation of placental nutrition at birth, and when exogenous substrate, such as intravenous glucose, is administered. ${ }^{12}$ Most infants achieve this homoeostasis, ${ }^{3}$ but metabolic adaptation after birth is deficient in some infants, resulting in hypoglycaemia or hyperglycaemia. Some authors maintain that insulin is the major glucoregulatory hormone in the neonatal period, ${ }^{4-6}$ but others argue for the importance of glucagon. ${ }^{7}$ Hepatic sensitivity to the action of these hormones is thought to differ between neonates and older subjects and to vary between subjects in the neonatal period. ${ }^{8-10}$ There are no studies linking blood glucose concentration, glucoregulatory hormone concentrations, and glucose production rates in healthy infants, but a recent study has shown that, while there are positive relationships between plasma insulin and blood glucose concentrations in healthy term and preterm infants, these relationships are different from those of older subjects in that, in neonates, high plasma insulin concentrations may be found when blood glucose concentrations are low. ${ }^{11}$

The aim of the present study was to demonstrate whether the mechanisms known to control glucose production in older subjects are defective in hypoglycaemic and hyperglycaemic infants. In particular, comparison of the insulin/glucose relationships for such infants with those of healthy infants ${ }^{11}$ would indicate whether disordered pancreatic insulin secretion contributed to hypoglycaemia or hyperglycaemia. Selected data from some of these hypoglycaemic infants have already been presented in a recent paper, ${ }^{12}$ but the interrelationships among blood glucose concentration, plasma insulin and glucagon concentration, and glucose production rate have not yet been described. This can be done most effectively by combining the data from the previous study with other (unpublished) data from hyperglycaemic infants, in order to analyse the relationships over a comprehensive range of blood glucose concentrations.

\section{Subjects and methods}

Ethical approval was granted by the ethics committee of Newcastle Health Authority, and parents gave informed consent for the inclusion of their babies in the study. During the study, the researchers did not alter the management already prescribed by the attending medical staff.

Twenty eight infants, of mixed gestation and less than 2 weeks old, who were undergoing investigation for hypoglycaemia or hyperglycaemia, were studied. Ten of these infants were hyperglycaemic (unpublished). Eighteen were receiving intravenous glucose infusion, 
prescribed by the attending medical staff as treatment for persistent hypoglycaemia, and for three of these infants hypoglycaemia was still present during the study, despite this treatment. ${ }^{12}$ The intravenous glucose infusion rate was not changed from that which had been prescribed by the attending clinician. No enteral feeds were given throughout the study period. Simultaneously with the prescribed glucose infusion, a primed, constant infusion of 6,6-di-deuterated glucose, enrichment $98 \%$, was infused (for hypoglycaemic infants, priming dose $=5.4 \mathrm{mg} / \mathrm{kg}$, infusion rate $=60 \mu \mathrm{g} / \mathrm{kg} / \mathrm{min}$; for hyperglycaemic infants, priming dose $=10.8 \mathrm{mg} / \mathrm{kg}$, infusion rate $120 \mu \mathrm{g} / \mathrm{kg} / \mathrm{min}$ ). The prescribed glucose infusion rate, and the tracer infusion rate, were not altered for at least one hour before sampling during the steady state period.

Blood samples (of $0.5 \mathrm{ml}$ each) were taken before the isotope infusion (time 0 ), and during the steady state period (times 60,75 , $90 \mathrm{~min}$ ) for subsequent measurement of plasma isotope enrichment. In addition, samples were collected during the steady state period (time $75 \mathrm{~min}$ ) for subsequent measurement of blood glucose and intermediary metabolite concentrations and plasma insulin and glucagon concentrations (total sample volume $1.5 \mathrm{ml}$ at time $75 \mathrm{~min}$ ). Blood for glucagon assay was collected into a tube containing aprotinin. Sample tubes were stored on ice during collection and plasma for hormone and isotope enrichment measurements was immediately separated and frozen at $-80^{\circ} \mathrm{C}$.

Blood glucose and intermediary metabolite concentrations and plasma insulin concentrations were assayed as described previously. ${ }^{3} 11$ Glucagon concentrations were measured in duplicate by radioimmunoassay, which had a sensitivity of $1.5 \mathrm{pmol} / 1$ and interassay coefficient of variation of $10 \% .^{13}$

A boroacetate derivative of glucose was prepared from plasma samples ${ }^{14}$ and the derivative was rediluted with ethyl acetate. Samples of $1 \mu \mathrm{l}$ were manually injected into a Finnigan Mat 1050 automated gas chromato- graphy/mass spectrometer (GC/MS) fitted with a DB5 capillary column. Ionisation was by electron impact. Ions 297 and 299 were monitored. Standard curves were obtained at the beginning and end of each run and the isotope enrichment of the infusate and plasma samples were determined.

Steady state was defined as occurring when the SD of the three isotope enrichment measurements (times 60, 75, and 90 minutes), was less than $10 \%$ of the mean of the measurements. As the rate of infusion of tracer (Rt) was known, the steady state equation (1) was used to calculate the glucose appearance rate (GAR). The infant's glucose production rate (GPR) was calculated by subtracting the known glucose intravenous (IV) infusion rate from the glucose appearance rate (2). ${ }^{15}$ The mean of the plasma isotope enrichment (IE) values was used in the calculation.

$$
\begin{gathered}
\text { GAR }=\frac{(\text { IE infusate }-1)}{(\text { IE plasma })} \times \text { Rt IE infusate }=98 \% \\
\text { GPR }=\text { GAR-IV glucose infusion rate }
\end{gathered}
$$

For each infant, a birthweight SD score was calculated, that is, the number of SDs the birth weight lay from the 50th centile for gestational age. The mid-arm circumference:head circumference (MAC:HC) ratio was calculated as a measure of asymmetry of growth retardation and the SD score calculated. ${ }^{16}$

Data were analysed using the SPSS-X package. Blood lactate, pyruvate, alanine, and glycerol concentrations $(\mathrm{mmol} / \mathrm{l})$ were summed to give total gluconeogenic substrate concentration. As blood glucose concentrations may reflect the balance between insulin and glucagon secretion, the insulin:glucagon ratio was calculated for each subject. As concentrations of insulin, glucagon, glucose and ketone bodies, and glucose appearance rate did not have normal distributions, nonparametric tests were used in the analysis, with Spearman correlation to assess the interrelationships between variables.

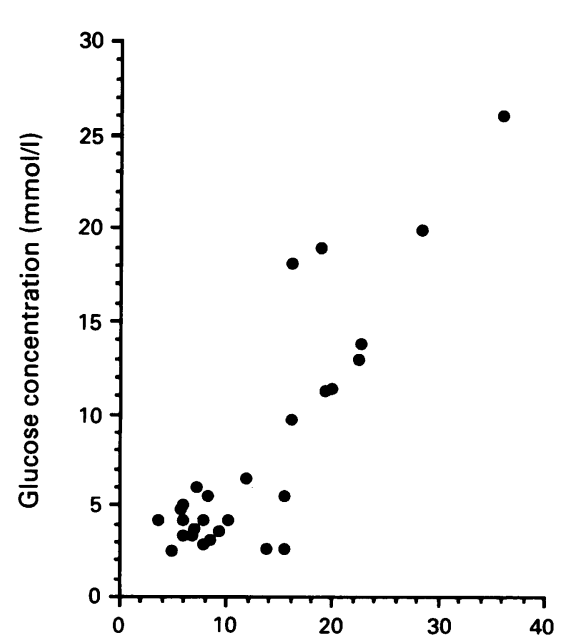

Glucose appearance rate $(\mathrm{mg} / \mathrm{kg} / \mathrm{min})$

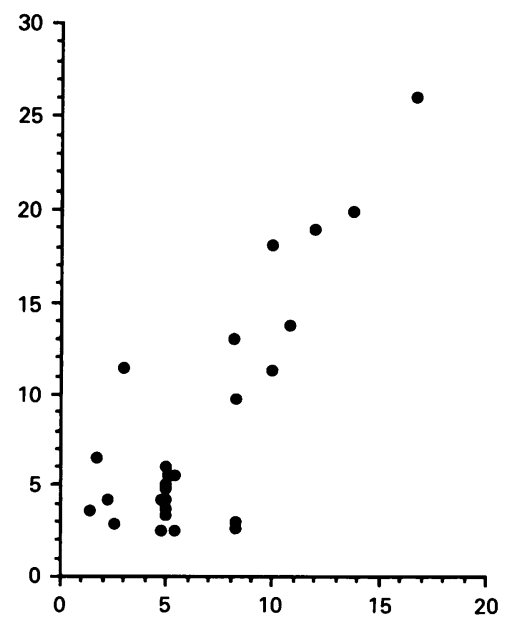

Glucose infusion rate $(\mathrm{mg} / \mathrm{kg} / \mathrm{min})$

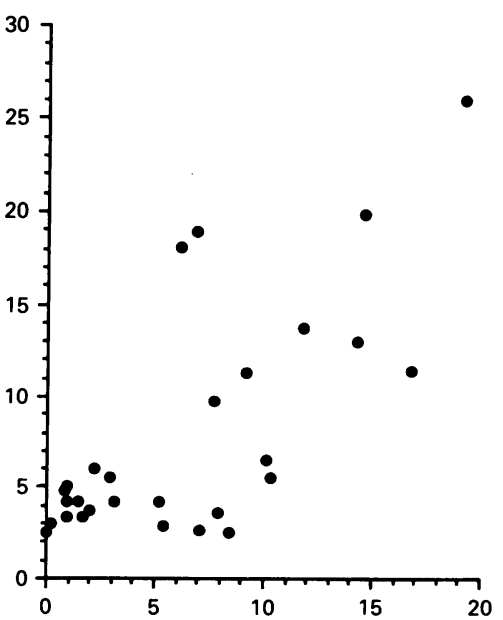

Glucose production rate $(\mathrm{mg} / \mathrm{kg} / \mathrm{min})$

Figure 1 The relationships of blood glucose concentration with glucose appearance rate, glucose infusion rate, and glucose production rate. 


\section{Results}

Details of the infants studied are shown in table 1. Most infants were small for gestational age, with the birth weight falling more than 2 SDs below the mean for gestational age, although the MAC:HC SD scores suggested that not all infants had asymmetrical growth retardation.

Blood glucose concentrations ranged from 2.5 to $26.1 \mathrm{mmol} / 1$. The infants with blood glucose concentrations above $7 \mathrm{mmol} / \mathrm{l}$ (hyperglycaemic infants) were preterm and of low birth weight. They were receiving intravenous glucose at a mean rate of $10 \cdot 2$ $\mathrm{mg} / \mathrm{kg} / \mathrm{min}$ (range $4 \cdot 4-16.7 \mathrm{mg} / \mathrm{kg} / \mathrm{min}$ ). The other infants who had been treated for hypoglycaemia received intravenous glucose at a mean rate of $4.8 \mathrm{mg} / \mathrm{kg} / \mathrm{min}$ (range $1-8 \mathrm{mg} / \mathrm{kg} / \mathrm{min}$ ).

Blood metabolite and hormone concentrations and glucose turnover data are shown in table 2 . Ketone body concentrations were low compared with healthy term infants, ${ }^{3}$ even at low blood glucose concentrations, and were not related to blood glucose concentrations nor to glucagon or insulin concentrations.

The relationships of blood glucose concentration with glucose appearance rate, glucose infusion rate, and glucose production rate are shown in fig 1. Blood glucose concentration was positively related to each of these parameters (for glucose appearance rate, $r=0.67$, $\mathrm{p}<0.001$; for glucose infusion rate, $r=0.51$, $\mathrm{p}<0.01$; for glucose production rate, $r=0.60$, $\mathrm{p}<0.001)$. All hyperglycaemic infants had glucose production rates above $5 \mathrm{mg} / \mathrm{kg} / \mathrm{min}$. There was a wide variation in glucose production rates for the 18 infants who were hypoglycaemic before or during the study $(0-8.4 \mathrm{mg} / \mathrm{kg} / \mathrm{min})$. There was a significant negative relationship between total gluconeogenic precursor concentration and glucose production rate $(r=-0.33, \mathrm{p}=0.043$; fig 2$)$.

The interrelationships between blood glucose concentration and plasma insulin and glucagon concentrations are shown in fig 3 . There was a weak positive correlation between blood glucose and plasma insulin concentrations $(r=0.38, p=0.025)$ but, especially at low

Table 1 Details of infants studied

\begin{tabular}{lll}
\hline & Median & Range \\
\hline Gestation (weeks) & 35 & $24-40$ \\
Birth weight (g) & 1695 & $670-3475$ \\
Birth weight SD score & $-2 \cdot 20$ & $-4 \cdot 66$ to $2 \cdot 11$ \\
MAC:HC SD score & $-0 \cdot 35$ & $-4 \cdot 96$ to $5 \cdot 14$ \\
Postnatal age (hours) & 41 & $6-240$ \\
\hline
\end{tabular}

Table 2 Metabolic data

\begin{tabular}{|c|c|c|}
\hline & Median & Range \\
\hline Glucose $(\mathrm{mmol} / \mathrm{l})$ & $5 \cdot 0$ & $2 \cdot 5-26 \cdot 1$ \\
\hline Total gluconeogenic & & \\
\hline substrate $(\mathrm{mmol} / \mathrm{l})$ & $2 \cdot 41$ & $1 \cdot 24-6 \cdot 71$ \\
\hline Ketone body $(\mathrm{mmol} / \mathrm{l})$ & 0.03 & $0.02-0.15$ \\
\hline Insulin $(\mathrm{mU} / \mathrm{l})$ & $4 \cdot 3$ & $<1 \cdot 0-45 \cdot 2$ \\
\hline Glucagon (pmol/l) & $22 \cdot 4$ & $5 \cdot 1-96 \cdot 2$ \\
\hline Insulin:glucagon ratio & 0.20 & $0.01-3.71$ \\
\hline Glucose appearance rate & & \\
\hline $\begin{array}{c}(\mathrm{mg} / \mathrm{kg} / \mathrm{min}) \\
\text { (n) }\end{array}$ & $9 \cdot 8$ & $3 \cdot 7-35 \cdot 9$ \\
\hline $\begin{array}{l}\text { Intravenous glucose } \\
(\mathrm{mg} / \mathrm{kg} / \mathrm{min})\end{array}$ & $5 \cdot 0$ & $1 \cdot 4-16 \cdot 7$ \\
\hline $\begin{array}{l}\text { Glucose production rate } \\
(\mathrm{mg} / \mathrm{kg} / \mathrm{min})\end{array}$ & $5 \cdot 8$ & $0 \cdot 0-19 \cdot 2$ \\
\hline
\end{tabular}

blood glucose concentrations, there was a wide range of insulin concentrations. There was a significant negative correlation between blood glucose and glucagon concentrations $(r=-0.62, \mathrm{p}<0.001)$; the glucagon concentrations are plotted on a logarithmic scale in fig 3 to demonstrate this correlation more clearly. There was a positive relationship between the

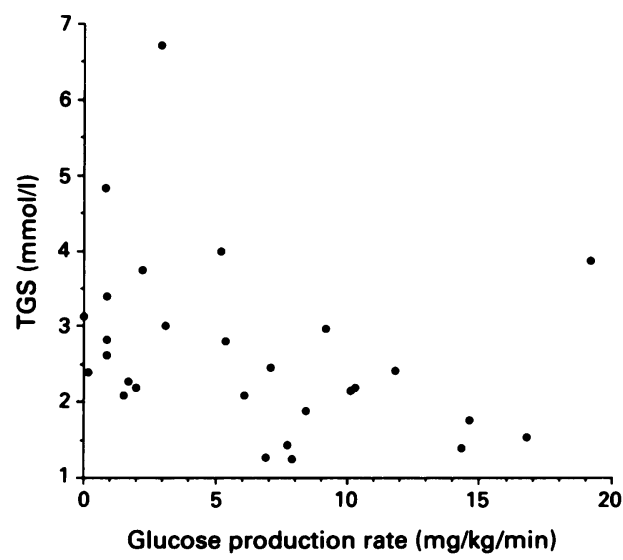

Figure 2 The relationship of blood total gluconeogenic substrate (TGS) concentration with glucose production rate.
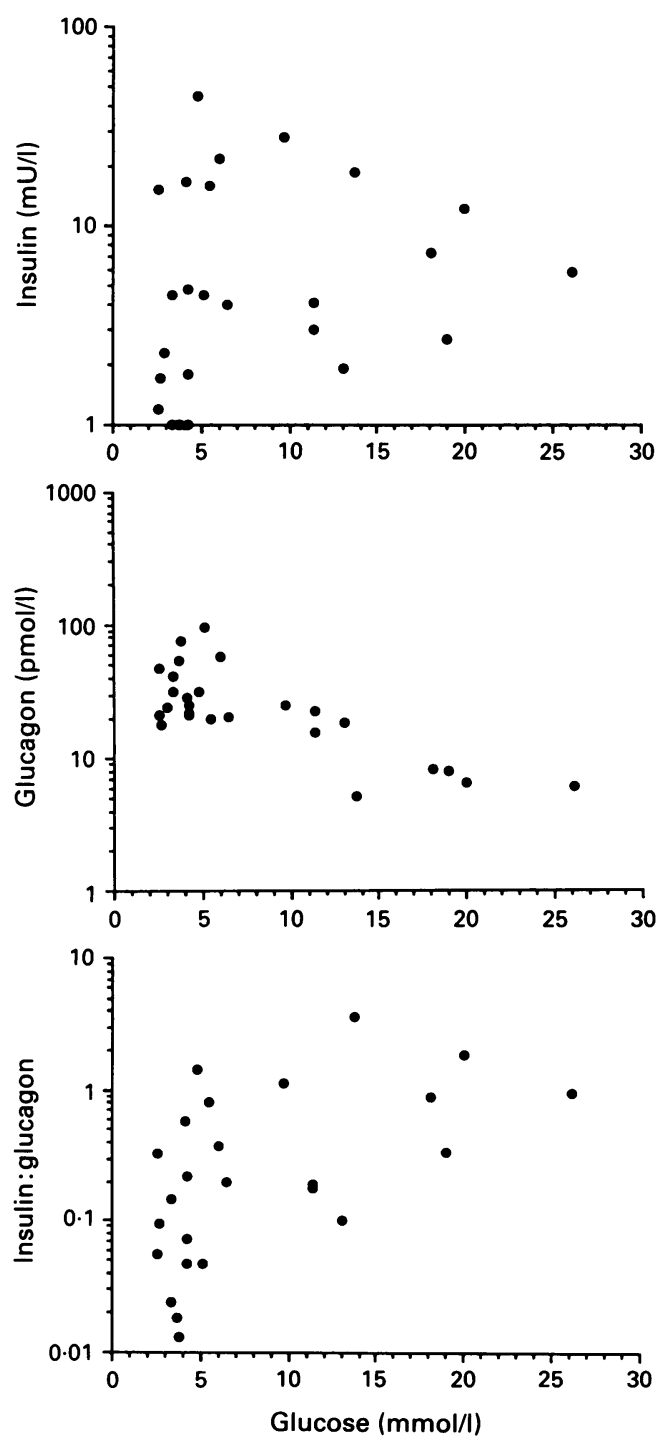

Figure 3 The relationships of plasma glucoregulatory hormone concentrations with blood glucose concentration. 

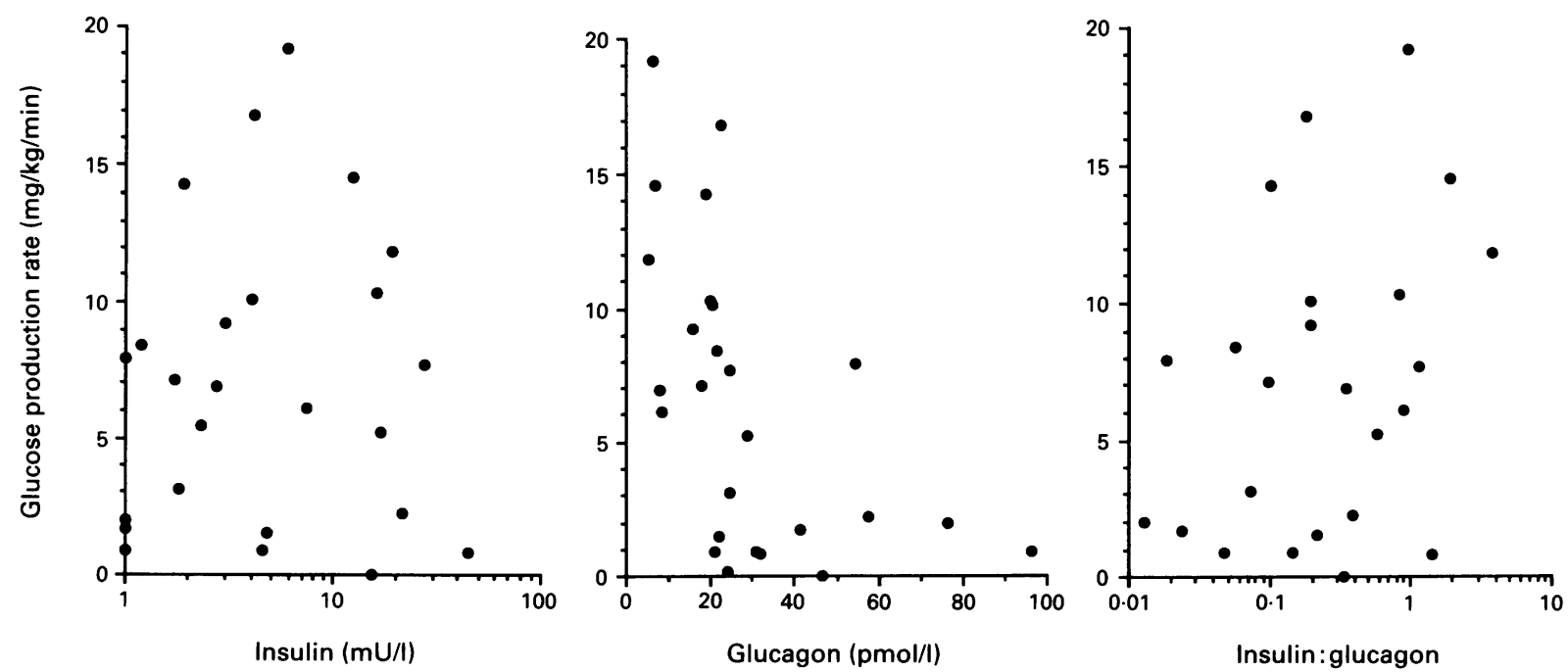

Figure 4 The relationships of glucose production rate with plasma glucoregulatory hormone concentrations.

insulin:glucagon ratio and blood glucose concentration $(r=0.60, \mathrm{p}<0.001)$.

Finally, the relationships of glucose production rate with plasma glucoregulatory hormone concentrations were considered (fig 4). A negative relationship between plasma glucagon concentration and glucose production rate was found $(r=-0.53, \mathrm{p}<0.01)$, but glucose production rate was not related to plasma insulin concentrations nor to the insulin:glucagon ratio.

\section{Discussion}

Previous studies of healthy term and preterm infants have demonstrated that endogenous glucose production rates, at $5-6 \mathrm{mg} / \mathrm{kg} / \mathrm{min}$, are higher than those of adults. ${ }^{17} 18$ Some infants suppress endogenous glucose production in response to increased glucose delivery, in the form of intravenous glucose infusion, ${ }^{19}$ while, in others, such suppression does not occur. ${ }^{2021}$ We have recently demonstrated the presence of a positive relationship between insulin and glucose concentrations in healthy term and preterm infants, although this relationship is less statistically significant than in older subjects. ${ }^{11}$ However, there have been no studies which document quantitative changes in glucose production rates in response to circulating blood glucose concentrations and glucoregulatory hormone concentrations.

We have demonstrated that in the group of infants studied there was disturbed autoregulation of blood glucose homoeostasis. This disordered homoeostasis was manifest as a failure to regulate glucose production rate in response to circulating blood glucose concentrations. Ethical constraints prevented the measurement of glucose production rates before the correction of hypoglycaemia with intravenous glucose, but our data suggest that hypoglycaemia may have been secondary to low glucose production rates in some infants, while hyperglycaemic infants had high glucose production rates. For autoregulation to occur, glucose production should have been enhanced at low blood glucose concentrations, and suppressed in the presence of hyper- glycaemia, to give a negative relationship between glucose production rate and glucose concentration, as opposed to the positive relationship demonstrated in this study.

There is evidence that this failure of autoregulation arose, first, from an inappropriate pancreatic endocrine secretory response and, second, as a result of insensitivity to the actions of these glucoregulatory hormones, at the level of hepatic gluconeogenesis.

In keeping with previous authors, we have demonstrated an extremely variable insulin secretory response to neonatal hypoglycaemia and hyperglycaemia, supporting the view that the $\beta$ cells of the fetal and neonatal pancreas appear to be relatively insensitive to circulating blood glucose concentrations. ${ }^{22} 23 \mathrm{~A}$ recent study of healthy infants has suggested that although there was an insulin secretory response to increasing blood glucose concentrations, this was extremely variable, particularly for preterm infants. ${ }^{11}$ Comparison of the data from this study (fig 3) and the previous study, ${ }^{11}$ demonstrate that the insulin/glucose relationship was even more variable for the infants with disordered blood glucose homoeostasis. Therefore, there appears to be a gradation of insulin response, there is a strong insulin/glucose relationship in mature subjects but this becomes weaker with decreasing maturity. Infants with disordered blood glucose homoeostasis may represent those with the most immature pancreatic insulin response. ${ }^{24}$

As in previous studies, however, the present study demonstrates that glucagon release was stimulated at low blood glucose concentrations and inhibited at high concentrations. ${ }^{25}$ The insulin:glucagon ratio, which reflects the balance between insulin and glucagon secretion was also variable, particularly at low blood glucose concentrations, but was raised in hyperglycaemic infants. Thus, in babies selected for study by virtue of their abnormalities of blood glucose homoeostasis, insulin secretion appeared to be less appropriate to the circulating blood glucose concentrations than glucagon secretion.

The glucose production rate in most subjects represents the sum of the processes of 
hepatic glycogenolysis and gluconeogenesis. The infants in the present study were unlikely to have substantial hepatic glycogen stores, by virtue of their immaturity or being small for gestational age, so that glucose production rate probably equalled the rate of hepatic gluconeogenesis from gluconeogenic precursors. Therefore it appears that hepatic gluconeogenesis was poorly controlled by the circulating pancreatic glucoregulatory hormone concentrations. We found that the glucose production rate did not have a negative relationship with the plasma insulin concentration nor the insulin:glucagon ratio, nor a positive relationship with the plasma glucagon concentration. These findings were the opposite of the expected relationships if the glucoregulatory hormones were adequately controlling hepatic glucose production. This suggests that neonatal hepatocytes were relatively insensitive to the actions of both glucoregulatory hormones. The hepatic insensitivity may be secondary to a postreceptor defect or to the action of other counterregulatory hormones, such as the catecholamines. Ethical and technical constraints prevented the collection of large enough blood samples to measure the concentrations of these hormones during the turnover studies.

The negative relationship between plasma glucagon concentrations and glucose production rates may be explained by the fact that the pancreatic glucagon secretory response to the circulating blood glucose concentrations was appropriate, but that gluconeogenesis was not affected by this glucagon response. Studies in animals support the hypothesis that an hepatic insensitivity to glucagon exists in neonatal subjects. ${ }^{26}$

The study demonstrates that the failure of gluconeogenesis was unlikely to be secondary to substrate deficiency. The negative relationship between blood total gluconeogenic substrate concentrations and glucose production rate may reflect the balance between glucagon stimulated total gluconeogenic substrate release at low blood glucose concentrations and the failure to utilise them for gluconeogenesis. Alternatively, utilisation of gluconeogenic substrates as alternative fuels may have a glucose sparing effect, so that rates of glucose production are not increased. Turnover studies with isotopically labelled substrate would be required to confirm this hypothesis.

As in previous studies, we have demonstrated that some infants mount a poor ketogenic response to low blood glucose concentrations. ${ }^{3} 27$ This may contribute to low rates of gluconeogenesis as the two processes are linked. ${ }^{28}$ In older subjects, both gluconeogenesis and ketogenesis are stimulated by glucagon and suppressed by insulin. ${ }^{29}$ However, we found no evidence that these hormones affected ketone body production in the infants studied. This observation may provide further evidence of hepatic insensitivity to these hormones. Alternatively, a deficiency of substrate in the form of free fatty acids, or cofactors such as carnitine, may have contributed to the low blood ketone body concentrations. Because of limitation of blood sample volumes we were unable to investigate this hypothesis by measuring these factors or by performing ketone body turnover studies.

Finally, we have demonstrated that blood glucose concentrations were closely related to intravenous glucose infusion rates. This is in agreement with previous studies. ${ }^{30}$ For infants at risk of failure of glucose autoregulation and of ketone body production, the clinician must be aware of the close relationship between the rate of glucose administration and blood glucose concentration. Blood glucose concentrations should be monitored if low or high administration rates are given, for example in fluid restricted babies or in those requiring high volume intakes to prevent dehydration. In addition, other mechanisms, such as enteral feeding, which appear to promote neonatal metabolic adaptation should be investigated, and, in terms of clinical management, policies of early enterally feeding should be encouraged whenever possible. ${ }^{3} 3132$

We thank the parents who gave consent for the inclusion of their babies in the study. We acknowledge the assistance of $M$ A McGann, Department of Child Health, Newcastle University, with metabolite assays; $\mathrm{Mr} \mathrm{C}$ Hetherington, Biomedical Mass Spectrometry Unit, Newcastle University, with turnover studies; $\mathrm{Dr} M$ Ghatei and Professor S Bloom, Hammersmith Hospital, London, with glucagon assays; and Ms L Ashworth and Professor KGGM Alberti, diabetic research group, department of medicine, Newcastle University, with insulin assays.

This work was presented in part at a meeting of the Pediatric Research Society, April, 1991

1 Blazquez E, Sugare M, Blazquez M, Fox PP. Neonatal changes in the concentration of liver CAMP and of serum glucose, FFA, insulin, pancreatic and total glucagon in man in the rat. $f$ Lab Clin Med glucagon in $1974 ; 83: 957-67$

2 Hetenyi G, Cowan JS. Glucoregulation in the newborn. Can $\mathcal{F}$ Physiol Pharmacol 1980;58:879-88.

3 Hawdon JM, Ward Platt MP, Aynsley-Green A. Patterns of metabolic adaptation for preterm and term infants in the first neonatal week. Arch Dis Child 1992;67:357-65.

4 Susa JB, Cowett RM, Oh W. Suppression of gluconeogenesis and endogenous glucose production by exogenous insulin administration in the newborn lamb. Pediatr Res 1979;13:594-9.

5 Cowett RM, Tenenbaum DG. Insulin is the primary hormone for control of neonatal glucose homeostasis. Pediatr Res 1985;19:311A.

6 Pildes RS, Pyati SP. Hypoglycaemia and hyperglycaemia in tiny infants. Clin Perinatol 1986;13:351-75.

7 Sperling MA. Integration of fuel homeostasis by insulin and glucagon in the newborn. Monographs in Pediatrics and glucagon 5 .

8 Sherwood WG, Hill DE, Chance GW. Glucose homeostasis in preterm monkey neonates. Pediatr Res 1977 11:874-7.

9 Pollack A, Cowett RM, Schwartz R. Glucose disposal in low birth weight infants during steady state hyperglycaemia: effects of exogenous insulin administration. Pediatrics 1978;61:546-9.

10 Cowett RM, Schwartz R. The role of hepatic control of glucose homeostasis in the aetiology of neonatal hypo and hyperglycaemia. Semin Perinatal 1979;3:327.

11 Hawdon JM, Aynsley-Green A, Alberti KGMM, Ward Platt MP. The role of pancreatic insulin secretion in neonatal glucoregulation. I. Healthy term and preterm infants. Arch Dis Child 1993;68:274-9.

12 Hawdon IM, Aynsley-Green A, Ward Platt MP. Neonata blood glucose concentrations: metabolic effects of intravenous glucagon and medium chain triglyceride. Arch Dis Child 1993;68:255-61.

13 Christofides ND. Pancreatic glucagon. In: Bloom SR Long $\mathrm{R}$, eds. Radioimmunoassay of gut regulatory peptides. Long R, eds. Radioimmunoassay

14 Weicko J, Sherman WR. Boroacetylation of carbohydrates. Correlations between structure and mass spectral Correlations between structure and mass spectral the American Chemical Society 1976;98:7631-7.

15 Wolfe RR. Tracers in metabolic research. Radioisotope and stable isotope mass spectrometry. Laboratory and research methods in biology and medicine. Vol 9 . New York: research methods in biology

16 Sasanow SR, Georgieff MK, Pereira GR. Mid-arm circumference and mid-arm circumference ratios: standard curves for anthropometric assessment of neonatal nutritional status. $\mathcal{F}$ Pediatr 1986;106:311-5. 
17 Bougneres PF. Stable isotope tracers and the determination of fuel fluxes in newborn infants. Biol Neonate 1987;52(suppl 1):87-96.

18 Bier DM, Leake RD, Haymond WH, et al. Measurement of true glucose production rates in infancy and childhood of true glucose production rates in infancy and childhood
with 6,6-di-deuteroglucose. Diabetes 1977;26:1016-23.

19 Kalhan SC, Oliven A, King KC, Lucero C. Role of glucose in regulation of endogenous glucose production in the human newborn Pediatr Res 1986;20:49-52.

20 Cowett RM, Oh W, Schwartz R. Persistent glucose production during glucose infusion in the neonate. $\mathcal{F}$ Clin Invest 1983;71:467-75

21 Cowett RM, Andersen GE, Maguire CA, Oh W. Ontogeny of glucose homeostasis in low birth weight infants. Pediatr 1988;112:462-5.

22 Grasso S, Messina A, Saporito W, Reitano G. Effect of theophylline plus glucagon on insulin secretion in the premature infant. Diabetes 1970;19:837-41.

23 Zarif M, Pildes RS, Vidyasagar D. Insulin and growth hormone responses in neonatal hyperglycaemia. Diabetes mone responses

24 Van Assche FA, Hoet JJ, Jack PMB. Endocrine pancreas of the pregnant mother, fetus and newborn. In: Beard RW, Nathanielsz PW, eds. Fetal physiology and medicine. New Nathanielsz PW, eds. Fetal physiology
York: Marcel Decker, 1984:127-52.

25 Grasso S, Fallucca F, Mazzone D, Giangrande L, Romeo
MG, Reitano G. Inhibition of glucagon secretion in the human newborn by glucose infusion. Diabetes 1983;32:489-92.

26 Bussey ME, Ogata ES, Finley S, La Barbera A Endogenous glucagon fails to induce hepatic phosphoenolpyruvate carboxykinase (PEPCK) in newborn growth enolpyruvate carboxykinase (PEPCK) in

retarded rats. Pediatr Res 1985;19:301A.
27 Hawdon JM, Ward Platt MP. Metabolic adaptation in small for gestational age infants. Arch Dis Child 1993; 68:262-8.

28 Girard J. Gluconeogenesis in the late fetal and early neonatal life. Biol Neonate 1986;50:237-58.

29 McGarry JD, Foster DW. Ketogenesis. In: Rifkin H, Porte $\mathrm{D}$, eds. Ellenberg and Rifkin's diabetes mellitus. Theory and practice. New York: Elsevier, 1990.

30 Zarlengo KM, Battaglia FC, Fenessey P, Hay WW. Relationship between glucose utilization rate and glucose concentration in preterm infants Biol Neonate 1986;49:181-9.

31 Sperling MA, De Lamater PV, Phelps D, Fiser D, Fisher DA. Spontaneous and amino acid stimulated glucagon secretion in the immediate postnatal period. 7 Clin Invest 1974;53:1159-66.

32 Lucas A, Bloom SR, Aynsley-Green A. Gastrointestinal peptides and the adaptation to extrauterine nutrition. Can f Physiol Pharmacol 1985;63:527-37. 